\title{
Pharmacokinetic interactions between ilaprazole and clarithromycin following ilaprazole, clarithromycin and amoxicillin triple therapy
}

\author{
Shan $\mathrm{CAO}^{1}$, Gan ZHOU ${ }^{1}$, Dong-sheng OU-YANG ${ }^{1}$, Hui-zi WU ${ }^{1}$, Kui XIAO ${ }^{2}$, Yao $\mathrm{CHEN}^{1}$, Dong GUO ${ }^{1}$, Lan FAN ${ }^{1}$, Zhi-rong TAN $^{1}$, \\ Hai-tang $\mathrm{HU}^{1}$, Xiang-hong $\mathrm{QIN}^{1}$, Hong-hao ZHOU ${ }^{1}$, Wei ZHANG ${ }^{1, *}$ \\ ${ }^{1}$ Institute of Clinical Pharmacology, Hunan Key Laboratory of Pharmacogenetics, Central South University, Changsha 410078, China; \\ ${ }^{2}$ Department of Respiratory Medicine, The Second Xiangya Hospital, Central South University, Changsha 410011, China
}

Aim: To investigate the drug interactions between ilaprazole, a new proton pump inhibitor, and clarithromycin following ilaprazole, clarithromycin and amoxicillin combination therapy.

Methods: Twelve healthy Chinese volunteers were recruited in a randomized, open-label, 3-period crossover study. All subjects were administered ilaprazole (5 mg), clarithromycin (500 mg) or a triple therapy, including ilaprazole (5 mg), clarithromycin (500 mg) and amoxicillin (1 g), twice daily for 6 consecutive days. On the 7th day, the drugs were given once, and blood samples were collected and analyzed using a well-validated HPLC/MS/MS method.

Results: Following the triple therapy, the peak concentration $\left(C_{\max }\right)$ and the area under the concentration-time curve from $0 \mathrm{~h}$ to $12 \mathrm{~h}\left(\mathrm{AUC}_{0 \rightarrow 12}\right)$ of ilaprazole were significantly decreased, as compared with the single medication group $\left(C_{\max }: 1025.0 \pm 319.6 \mathrm{vs}\right.$ $1452.3 \pm 324.6 \mathrm{ng} / \mathrm{mL} ; A_{U C} C_{0 \rightarrow 12}: 9777.7 \pm 3789.8$ vs $\left.11363.1 \pm 3442.0 \mathrm{ng} \cdot \mathrm{h} / \mathrm{mL}\right)$. Similar changes were found for ilaprazole sulfone $\left(C_{\max }: 5.9 \pm 0.5\right.$ vs $9.3 \pm 1.7 \mathrm{ng} / \mathrm{mL} ; \mathrm{AUC}_{0 \rightarrow 12}: 201.4 \pm 32.1$ vs $\left.277.1 \pm 66.2 \mathrm{ng} \cdot \mathrm{h} / \mathrm{mL}\right)$. The triple therapy significantly elevated the $C_{\max }$ of clarithromycin (3161.5 \pm 702.2 vs $2541.9 \pm 476.2 \mathrm{ng} / \mathrm{mL})$.

Conclusion: The $\mathrm{H}$ pylori eradication therapy with clarithromycin, amoxicillin and ilaprazole may cause pharmacokinetic interactions that decrease the amount of ilaprazole and its metabolites and elevate that of clarithromycin.

Keywords: peptic ulcer; Helicobacter pylori infection; eradication therapy; clarithromycin; amoxicillin; ilaprazole; pharmacokinetics; drug interaction

Acta Pharmacologica Sinica (2012) 33: 1095-1100; doi: 10.1038/aps.2012.64; published online 23 Jul 2012

\section{Introduction}

Seventy to $90 \%$ of all peptic ulcer disease cases, one of the most common diseases influencing health around the world, are caused by Helicobacter pylori (H pylori) infection ${ }^{[1]}$. The choice of a treatment regimen for $H$ pylori-positive cases is based on the principle of infection eradication ${ }^{[2]}$. With single antibiotic treatment, the eradication rate achieved is between $15 \%$ and $54 \%$ when the macrolide antibiotic, clarithromycin (CLR), is given ${ }^{[3]}$ and is between $20 \%$ to $30 \%$ when the penicillin antibiotic, amoxicillin (AMX), is administrated ${ }^{[4]}$. A triple therapy comprised of a proton pump inhibitor (PPI), such as omeprazole, lansoprazole or rabeprazole, with AMX and CLR has been strongly recommended as a first-line therapy for $H$

\footnotetext{
* To whom correspondence should be addressed.

E-mail yjsd2003@163.com

Received 2012-02-02 Accepted 2012-05-07
}

pylori eradication ${ }^{[5]}$.

Previous reports indicate that following triple therapy, the rate of $H$ pylori eradication achieved was $84 \%-95 \%{ }^{[4,6]}$. The synergistic effect of these therapies is related to pharmacokinetic interactions. It has been confirmed that triple therapy increases both the AUC and $C_{\max }$ of clarithromycin and its active metabolite $14-\mathrm{OH}$-clarithromycin ${ }^{[7-9]}$. However, the AUC of omeprazole increased almost 2-fold after concomitant administration of CLR ${ }^{[7]}$, and the AUC of lansoprazole significantly increased from 3.65 to $4.59 \mathrm{mg} \cdot \mathrm{h} / \mathrm{L}$ after co-administration of CLR and AMX ${ }^{[8]}$. The pharmacokinetic drug interactions are likely a result of alterations in the hepatic metabolism of PPIs following combination therapy with clarithromycin, which is considered a potent inhibitor of CYP3A ${ }^{[10-12]}$. Ushiama et al previously published data using partial cortisol clearance as a CYP3A activity probe in vivo to assess the inhibition of CYP3A activity. In their study, two experimental 
groups were given clarithromycin (400 mg or $800 \mathrm{mg}$ ), and they observed a dose-dependent inhibition of in vivo CYP3A activity and resultant elevation in plasma lansoprazole concentrations, which were not present in the control group that did not receive clarithromycin ${ }^{[13]}$.

A new proton pump inhibitor, ilaprazole (Ila), was recently introduced. In a double-blind, parallel, randomized study, ilaprazole was shown to be as effective and well-tolerated as omeprazole for the treatment of gastric and duodenal ulcers (healed in $67.1 \%$ vs $62.3 \%$ and $83.7 \%$ vs $78.9 \%$ of patients, respectively), at a much lower dose (5 vs $20 \mathrm{mg}$, respectively ${ }^{[14]}$. In another multicenter trial, $10 \mathrm{mg}$ ilaprazole was found to be more effective than $20 \mathrm{mg}$ omeprazole and other doses of ilaprazole over a 4-week treatment period ${ }^{[15]}$.

A study on rat livers using on-line HPLC/ESI mass spectrometry found two metabolites of ilaprazole - a major product, ilaprazole sulfone, and a minor product, hydroxyl-ilaprazole $^{[16]}$ - implying that ilaprazole might be predominantly metabolized in the liver by CYP3A and partially by CYP2C19 in a manner similar to that of omeprazole and lansoprazole, which are metabolized into sulfoxidation and hydroxylation metabolites. However, data published by $\mathrm{Li}$ and Cho et al indicate that CYP3A5 and CYP2C19 genotypes have no impact on ilaprazole metabolism ${ }^{[17,18]}$. Therefore, further investigation is required to determine whether pharmacokinetic interactions play a role in triple therapy using ilaprazole as a PPI.

Although its pharmacological interaction mechanisms are not fully understood, triple therapy has been used for many years in the treatment of $H$ pylori-related peptic ulcer disease. Our study use ilaprazole as a novel PPI within a triple therapy regimen. The present study was designed to determine the effect of (1) clarithromycin and amoxicillin on the pharmacokinetics of ilaprazole and its metabolites and (2) ilaprazole and amoxicillin on the pharmacokinetics of clarithromycin.

\section{Materials and methods}

\section{Materials and reagents}

Ilaprazole, ilaprazole sulfone and ilaprazole thiol ether standards were obtained from the Livzon Pharmaceutical Group, Inc (purity: 99.1\%, Zhuhai, China). Omeprazole standards were obtained from Sigma Chemical Company (purity: 99.5\%, USA). Ilaprazole tablets were obtained from the Livzon Pharmaceutical Group, Inc (Zhuhai, China). HPLC-grade acetonitrile, methanol and methyl tert-butyl ether (MTBE) were purchased from Dikma Comp (Guangzhou, China). HPLCgrade water was obtained using a Milli-Q system (Millipore, USA). All other reagents were of analytical grade.

\section{Subjects}

This study was conducted under the approval of the Xiangya institutional review boards and with the informed consent of all the subjects. Twelve healthy, Chinese Han male volunteers with normal hepatic, renal, and hematologic function were selected for this study. All results were within the normal limits (see Table 1S). The age of the participants ranged from 21 to 27 years (mean $\pm S D, 23.9 \pm 1.9$ years), their weight ranged from 55 to $77 \mathrm{~kg}(63.7 \pm 7.8 \mathrm{~kg})$, and their height ranged from 162 to $178 \mathrm{~cm}(172.2 \pm 4.2 \mathrm{~cm})$. The exclusion criteria included any use of medication within 2 weeks prior to the commencement of the study, the use of tobacco or alcohol during the study, and any clinically significant illness within 3 months prior to the study. A physical examination was performed, and common laboratory parameters for hematological, hepatic and renal functions were determined, before, during and after the study to monitor the safety of drug treatment.

\section{Study protocol}

This was a randomized, open-labeled, self-controlled, 3-way crossover study. Twelve subjects were randomized into 3 groups: ilaprazole $(5 \mathrm{mg})$; clarithromycin $(500 \mathrm{mg})$; or ilaprazole $(5 \mathrm{mg})$, clarithromycin $(500 \mathrm{mg})$ and amoxicillin $(1 \mathrm{~g})$ combination therapy. All drugs were administered twice daily for 6 consecutive days and only once on the 7 th $\mathrm{d}$. After $12 \mathrm{~h}$ of fasting, the volunteers were given the drugs with $200 \mathrm{~mL}$ tap water on the morning of the $7 \mathrm{th} \mathrm{d}$. Then, $5 \mathrm{~mL}$ or $8 \mathrm{~mL}$ blood samples were collected before and $0.5,1,1.5,2,3,4,6,8,12,24$, 48 , and $72 \mathrm{~h}$ after drug administration. The whole blood sample was centrifuged immediately, and the upper layer serums were transferred to polyethylene tubes and stored at $-80^{\circ} \mathrm{C}$ until analysis. After a 7-d drug elimination period between each dose, the subjects proceeded to the next phase.

\section{Measurement of serum drug concentration}

The quantitative determination of ilaprazole and its two metabolites was based on an improved LC/MS/MS method published by Zhou et al ${ }^{[16,19]}$. Briefly, the chromatographic condition included a Thermo HyPURITY C18 column (150 $\mathrm{mm} \times 2.1 \mathrm{~mm}, 5 \mu \mathrm{m}$ ) with a mobile phase consisting of 10 $\mathrm{mmol} / \mathrm{L}$ ammonium formate water-acetonitrile solution (50:50, $v / v)$ at a flow rate of $0.25 \mathrm{~mL} / \mathrm{min}$. The API4000 triple quadruple mass spectrometer (Applied Biosystems, USA) was operated in multiple reactions monitoring mode via positive electrospray ionization interface.

This method displayed linearity over the concentration range of $0.23-2400.00 \mathrm{ng} / \mathrm{mL}$ for ilaprazole, $0.05-105.00$ $\mathrm{ng} / \mathrm{mL}$ for ilaprazole thiol ether and $0.06-45.00 \mathrm{ng} / \mathrm{mL}$ for ilaprazole sulfone. The lower limits of quantification were $0.23,0.05$, and $0.06 \mathrm{ng} / \mathrm{mL}$ for ilaprazole, ilaprazole sulfone and ilaprazole thiol ether, respectively. The intra- and interday precisions were all less than $15 \%$ in terms of relative standard deviation (RSD), and the accuracy was within 15\% in terms of relative error (RE) for ilaprazole, ilaprazole sulfone and ilaprazole thiol ether.

The concentration of clarithromycin in plasma was determined using HPLC-MS/MS. Separation of the analytes and the internal standard (IS), roxithromycin, was performed on a Waters Xterra MS C18 column $(2.11 \mathrm{~mm} \times 50 \mathrm{~mm}, 5 \mu \mathrm{m})$, with a mobile phase consisting of $20 \mathrm{mmol} / \mathrm{L}$ ammonium acetate water-acetonitrile solution $(40: 60, v / v)$, at a flow rate of 0.25 $\mathrm{mL} / \mathrm{min}$. The API4000 triple quadruple mass spectrometer was operated in multiple reactions monitoring mode via a positive electrospray ionization interface. The standard 
curves displayed excellent linearity with no systematic bias. The lower limit of quantification of clarithromycin was 6.035 $\mathrm{ng} / \mathrm{mL}$, the intra- and inter-day precisions were all less than $15 \%$ for medium and high concentrations and less than $20 \%$ for low concentration in terms of the relative standard deviation (RSD), and the accuracy was within 15\% in terms of the relative error (RE) for clarithromycin at all concentrations.

\section{Pharmacokinetic analysis}

Pharmacokinetic parameters were calculated according to a non-compartmental model using Winnonlin (Pharsight Corporation, ver 3.0, Mountain View, CA, USA). The peak concentration $\left(C_{\max }\right)$ and the time of peak concentration $\left(T_{\max }\right)$ were directly analyzed by visual inspection of the plasma concentration-time profile. The elimination rate constant $(\lambda)$ was obtained by the least square fitted terminal log-linear portion of the slope of the plasma concentration-time profile, and the elimination half-life $\left(t_{1 / 2}\right)$ was evaluated according to $0.693 / \lambda$. The area under the plasma concentration-time curve $\left(\mathrm{AUC}_{\infty}\right)$ was evaluated by the linear trapezoidal rule and further extrapolated to infinity by dividing the last measurable concentration by $\lambda$, according to the following equation: $\mathrm{AUC}_{\infty}=\mathrm{AUC}_{0-\mathrm{t}}+\mathrm{C}_{\text {last }} / \lambda$.

\section{Statistical analysis}

All data are expressed as the mean \pm SD. Statistical comparisons of the mean values between different groups were performed using a paired $t$-test or an AVONA. The data were analyzed using the statistical program SPSS (Statistical Package for the Social Sciences) 11.0 for Windows (SPSS, Chicago, IL, USA). Differences were considered statistically significant at $P<0.05$. The $90 \%$ confidence intervals of the log-values of the AUC and $C_{\max }$ were determined for the bio-equivalence test. The resulting data beyond $70 \%-143 \%$ for $C_{\max }$ and $80 \%-$ $125 \%$ for AUC in single treatment groups were considered significantly different.

\section{Results}

All 12 of the volunteers completed the 3 phases of the study under the well-performed protocol. No severe adverse events or clinically relevant deviations of laboratory parameters occurred during the study.

The pharmacokinetic interaction effect of triple therapy on clarithromycin or ilaprazole and its metabolites (ilaprazole sulfone and ilaprazole thiol-ether) were investigated by comparing the concentration and kinetic parameters of the single administration cycle with combination therapy. The pharmacokinetic parameters for the indicated drugs and ilaprazole metabolites after single and triple drug therapy are summarized in Table 1, and serum concentration-time profiles are presented in Figure 1.

The maximum concentration $\left(C_{\max }\right)$ of ilaprazole was significantly decreased after combination therapy compared with single treatment $(1025.0 \pm 319.6$ vs $1452.3 \pm 324.6$ $\mathrm{ng} / \mathrm{mL}, P=0.002)$. The $\mathrm{AUC}_{0 \rightarrow 12}$ and $\mathrm{AUC}_{0 \rightarrow \infty}$ of ilaprazole were $9777.7 \pm 3789.8$ and $9870.5 \pm 3910.7 \mathrm{ng} \cdot \mathrm{h} / \mathrm{mL}$ in the triple therapy group, which were much lower than the corresponding values in the single treatment group (11363.1 \pm 3442.0 and $11438.8 \pm 3473.5 \mathrm{ng} \cdot \mathrm{h} / \mathrm{mL}$, respectively), but the differences did not reach statistical significance.

Following triple therapy, the mean residence time (MRT) and the total clearance/systemic bioavailability $\left(\mathrm{CL}_{\text {tot }} / f\right)$ of ilaprazole increased compared to the single treatment period, whereas the elimination half-life $\left(t_{1 / 2}\right)$ decreased slightly.

In the triple therapy group, the $90 \%$ confidence intervals (CIs) of $C_{\text {max }}, \mathrm{AUC}_{0 \rightarrow 12}$, and $\mathrm{AUC}_{0 \rightarrow \infty}$ were $58.3 \%$ to $80.5 \%$, $70.7 \%$ to $99.9 \%$, and $70.7 \%$ to $100.1 \%$ (Table 2), respectively, which represents a decrease of $24.9 \%, 8.3 \%$, and $8.2 \%$ compared with the corresponding values in the single treatment group, respectively. All of these data fell completely beyond the equivalence range of $80 \%-125 \%$ for AUC and $70 \%-143 \%$ for $C_{\max }$.

The changes tendency of pharmacokinetic parameters of

Table 1. Pharmacokinetic parameters of ilaprazole (Ila) and its metabolites (Sulfone and Thiol ether), clarithromycin (CLR), following single and ilaprazole clarithromycin and amoxicillin (AMX) triple therapy. Mean \pm SD. $n=12 .{ }^{\mathrm{b}} P<0.05$.

\begin{tabular}{|c|c|c|c|c|c|c|c|}
\hline Treatment & $\begin{array}{l}T_{1 / 2} \\
\text { (h) }\end{array}$ & $\begin{array}{c}C_{\max } \\
(\mathrm{ng} / \mathrm{mL})\end{array}$ & $\begin{array}{l}T_{\max }{ }^{*} \\
(\mathrm{~h})\end{array}$ & $\begin{array}{c}\mathrm{AUC}_{(0 \rightarrow t)} \\
(\mathrm{ng} \cdot \mathrm{h} / \mathrm{mL})\end{array}$ & $\begin{array}{l}\operatorname{AUC}_{(0 \rightarrow \infty)} \\
(\mathrm{ng} \cdot \mathrm{h} / \mathrm{mL})\end{array}$ & $\begin{array}{l}\text { MRT } \\
\text { (h) }\end{array}$ & $\begin{array}{l}\mathrm{CL} / \mathrm{F} \\
(\mathrm{L} / \mathrm{h})\end{array}$ \\
\hline Ila & $10.1 \pm 3.2$ & $1452.3 \pm 324.6$ & $3(2-4)$ & $11363.1 \pm 3442.0$ & $11438.8 \pm 3473.5$ & $9.8 \pm 3.0$ & $0.4 \pm 0.2$ \\
\hline Ila+AMX+CLR & $9.3 \pm 3.7$ & $1025.0 \pm 319.6^{b}$ & $3(1.5-6)$ & $9777.7 \pm 3789.8^{b}$ & $9870.5 \pm 3910.7^{b}$ & $10.1 \pm 2.9$ & $0.5 \pm 0.2$ \\
\hline Sulfone (Ila) & $19.1 \pm 9.1$ & $9.3 \pm 1.7$ & $4(0.5-8)$ & $277.1 \pm 66.2$ & $308.0 \pm 95.6$ & $21.6 \pm 3.1$ & \\
\hline Sulfone (Ila+AMX+CLR) & $20.8 \pm 6.0$ & $5.9 \pm 0.5^{b}$ & $6(0.5-12)$ & $201.4 \pm 32.1^{b}$ & $224.7 \pm 47.7^{b}$ & $22.7 \pm 2.5$ & \\
\hline Thiol ether (Ila) & $11.9 \pm 7.2$ & $15.7 \pm 7.1$ & $4(1.5-6)$ & $246.0 \pm 178.3$ & $272.8 \pm 214.5$ & $13.6 \pm 5.3$ & \\
\hline Thiol ether (Ila+AMX+CLR) & $13.1 \pm 6.1^{\mathrm{b}}$ & $16.0 \pm 7.7$ & $3(0.5-6)^{b}$ & $268.4 \pm 220.9$ & $279.3 \pm 220.5$ & $17.6 \pm 9.4^{\mathrm{b}}$ & \\
\hline CLR & $10.9 \pm 2.9$ & $2541.9 \pm 476.2$ & $1.5(1.5-2)$ & $29772.7 \pm 4781.9$ & $30254.9 \pm 5240.4$ & $12.8 \pm 2.6$ & $16.5 \pm 3.0$ \\
\hline$C L R+A M X+11 a$ & $8.5 \pm 1.4$ & $3161.5 \pm 702.2^{b}$ & $1.5(1.5-4)$ & $29953.4 \pm 6474.4$ & $30045.5 \pm 6490.6$ & $10.4 \pm 1.2^{b}$ & $16.6 \pm 4.2$ \\
\hline
\end{tabular}

${ }^{*} \mathrm{~T}_{\max }$ was described by median (range). 

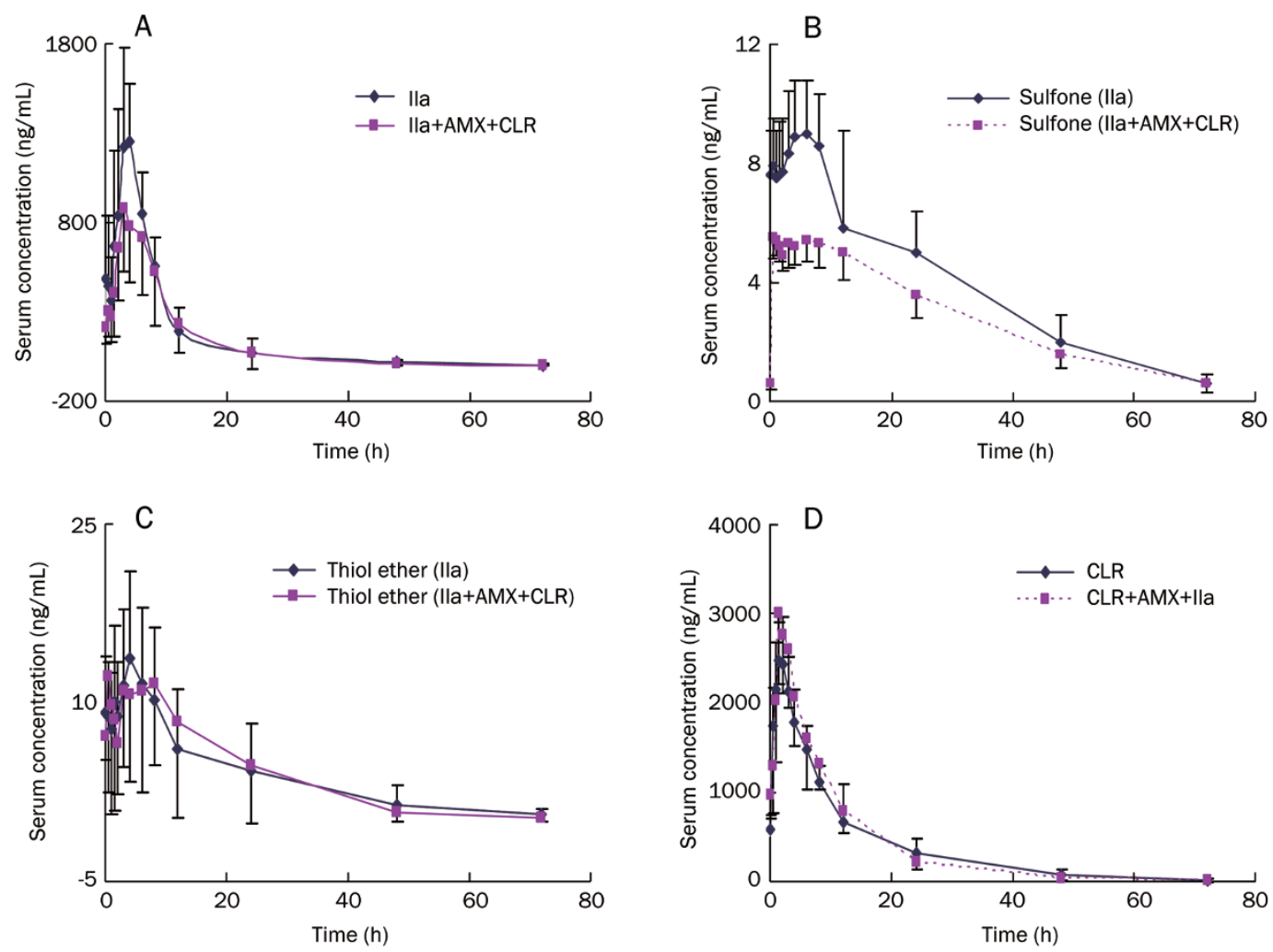

Figure 1. Serum concentrations of ilaprazole (A), ilaprazole sulfone (B), ilaprazole thiolether (C), clarithromycin (D), expressed as mean $\pm S D$ and regression line after administration of ilaprazole alone and with amoxicillin and clarithromycin. Ila, ilaprazole; AMX, amoxicillin; CLR, clarithromycin.

Table 2. Test of bio-euivalence after administration of ilaprazole alone and with amoxicillin and clarithromycin.

\begin{tabular}{|c|c|c|c|}
\hline \multirow[b]{2}{*}{ Treatment } & \multicolumn{3}{|c|}{ Point estimates ( $90 \%$ confidence interval) } \\
\hline & $A \cup C_{0 \rightarrow t}$ & $A \cup C_{0 \rightarrow \infty}$ & $C_{\max }$ \\
\hline Ila & $81.4(70.7-99.9)^{a}$ & $84.1(70.7-100.1)^{\mathrm{a}}$ & $68.5(58.3-80.5)^{a}$ \\
\hline \multicolumn{4}{|l|}{$\| \mathrm{Ia}+\mathrm{AMX}+\mathrm{CLR}$} \\
\hline Sulfone & $80.4(69.4-96.4)^{a}$ & $83.5(69.5-96.4)^{a}$ & $66.3(58.4-77.2)^{a}$ \\
\hline \multicolumn{4}{|c|}{ Sufone (Ila+AMX+CLR) } \\
\hline CLR & $99.5(81.5-121.5)$ & $98.4(80.1-120.9)$ & $122.6(100.7-149.2)^{a}$ \\
\hline $\mathrm{CLR}+\mathrm{AMX}+\| \mathrm{la}$ & & & \\
\hline
\end{tabular}

${ }^{\text {a }}$ Point estimates $(90 \%$ confidence interval) in triple therapy beyond the set value in single therapy.

ilaprazole sulfone, the major ilaprazole metabolite, was similar to that of its parent drug, ilaprazole. We observed a large difference in the $C_{\max }$ values between the triple therapy group $(5.9 \pm 0.5 \mathrm{ng} / \mathrm{mL})$ and the single therapy group $(9.3 \pm 1.7 \mathrm{ng} / \mathrm{mL}$; $\left.P=2.25 \times 10^{-5}\right)$. A smaller difference was observed between the $\mathrm{AUC}_{0 \rightarrow 12}$ and $\mathrm{AUC}_{0 \rightarrow \infty}$ of ilaprazole sulfone following triple therapy and single therapy $(201.4 \pm 32.1$ vs $277.1 \pm 66.2 \mathrm{ng} \cdot \mathrm{h} / \mathrm{mL}$, $P=1.46 \times 10^{-9}$ and $224.7 \pm 47.7 \mathrm{ng} \cdot \mathrm{h} / \mathrm{mL}$ vs $308.0 \pm 95.6 \mathrm{ng} \cdot \mathrm{h} / \mathrm{mL}$, $P=2.36 \times 10^{-8}$, respectively). In the triple therapy group, the $90 \%$ CIs of $C_{\text {max }}, \mathrm{AUC}_{0 \rightarrow 12}$ and $\mathrm{AUC}_{0 \rightarrow \infty}$ were significantly decreased by $30.4 \%, 22.1 \%$, and $21.3 \%$ compared with the corresponding values in the single treatment group (Table 2).

For ilaprazole thiol ether, the $T_{\max }$ was reached at $3 \mathrm{~h}$ (median, range 0.5-6 h) after combination treatment, which was significantly faster than that of single treatment, which was reached at $4 \mathrm{~h}$ (median, range 1.5-6 h) $(P=0.009)$. A small increase was observed in the $C_{\max }$ of the combination treatmeant group $(16.0 \pm 7.7 \mathrm{ng} / \mathrm{mL})$ compared with the single therapy group $(15.7 \pm 7.1 \mathrm{ng} / \mathrm{mL})$, but this difference was not significant. A slight increase was observed in $\mathrm{AUC}_{0 \rightarrow 12}$ and $\mathrm{AUC}_{0 \rightarrow \infty}$ after combination therapy compared to single therapy (268.4 \pm 220.9 vs $246.0 \pm 178.3 \mathrm{ng} \cdot \mathrm{h} / \mathrm{mL}, P=0.728 ; 279.3 \pm 220.5$ vs 
$272.8 \pm 214.5 \mathrm{ng} \cdot \mathrm{h} / \mathrm{mL}, P=0.736$, respectively). The $90 \%$ CIs of $\mathrm{AUC}_{0 \rightarrow 12}$ and $\mathrm{AUC}_{0 \rightarrow \infty}$ in the combination treatment group were beyond $80 \%-125 \%$, and the $C_{\max }$ was within the $70 \%-$ $143 \% \mathrm{CI}$ range in the single treatment group.

For clarithromycin, a $29.8 \%$ increase in $C_{\max }$ was measured after combination treatment compared with single treatment (3161.5 \pm 702.2 vs $2541.9 \pm 476.2 \mathrm{ng} / \mathrm{mL}, P=0.0089$. The $90 \%$ CIs of $C_{\max }$ following the three-drug regimen was beyond the range of $70 \%-143 \%$, and the other parameters were altered slightly without any statistical significance.

\section{Discussion}

No significant positive synergistic effect was found between ilaprazole, clarithromycin and amoxicillin in this study. Unexpectedly, when combined with clarithromycin and amoxicillin, the plasma concentrations of ilaprazole were lower than those of single ilaprazole administration over a 6-day course of therapy. The mean $C_{\max }, \mathrm{AUC}_{0 \rightarrow 12}$, and $\mathrm{AUC}_{0 \rightarrow \infty}$ were decreased by $24.9 \%, 8.3 \%$, and $8.2 \%$, respectively, when ilaprazole was given in combination compared to its administration as a single agent. The significant decrease in $C_{\max }$, the decreased AUC and the lack of alteration in the $T_{1 / 2}$ of ilaprazole in serum indicate that the reduced plasma concentrations observed in our study were likely due to diminished absorption as opposed to alterations in metabolism.

The significant decrease in the $C_{\max }$ and AUC of ilaprazole sulfone after triple therapy support this consideration. The same trend was considered to be due to decreased absorption of the parent drug; however, the AUC of ilaprazole thiol ether did not change after triple therapy, which supports Zhou's report demonstrating that it is a relatively minor metabolite ${ }^{[16]}$.

Early studies investigated the effect of clarithromycin - an accepted, potent inhibitor of CYP3A - on the pharmacokinetics of PPIs because in vivo and in vitro studies suggested that PPIs, such as omeprazole and lansoprazole, are metabolized, at least in part, by CYP3A4. It was previously demonstrated that the plasma exposure of omeprazole and lansoprazole increased almost 2-fold ${ }^{[7,13]}$ and $1.3-$ fold $^{[8]}$, respectively, when given simultaneously with clarithromycin. Compared with omeprazole, esomeprazole metabolism is more dependent on CYP3A. One study indicated that clarithromycin decreased the rate of esomeprazole metabolism, which doubled the AUC values, regardless of the CYP2C19 genotype $^{[9]}$. The pharmacokinetics of rebeprazole, which is metabolized by a non-enzymatic pathway with minor CYP2C19 and CYP3A4 involvement, were not altered by clarithromycin or verapamil (a potent CYP3A inhibitor), irrespective of the CYP2C19 genotype ${ }^{[20]}$.

For ilaprazole, $\mathrm{Li}$ and Cho reported that the CYP3A5 and CYP2C19 genotypes had no impact on ilaprazole metabolism in healthy Chinese and Korea subjects ${ }^{[17,18]}$. Additionally, our previous study evaluating the effect of the CYP3A phenotype (indicated by $1-\mathrm{OH}$ midazolam/midazolam ratio) on ilaprazole metabolism showed no correlation between the CYP3A phenotype and ilaprazole metabolism. The reduced influence of CYP3A on ilaprazole metabolism may explain why ilapra- zole and clarithromycin did not produce synergistic effects in our study. To date, no transporters involved in ilaprazole absorption and metabolism have been identified. The reason for the decreased absorption of ilaprazole after triple therapy warrants further investigation.

It is well established that amoxicillin is primarily excreted as a pro-drug and is not metabolized in the liver. Mainz reported that the pharmacokinetics of amoxicillin were not altered by combination therapy with lansoprazole and clarithromycin ${ }^{[8]}$, which is consistent with other reports that demonstrated no effects of omeprazole on the pharmacokinetics of amoxicil$\operatorname{lin}^{[21-23]}$. Therefore, in present study, we did not compare the plasma concentration of amoxicillin alone with combination therapy because of its non-metabolic characteristics.

In this study, the pharmacokinetic parameters of clarithromycin were comparable with those of other studies ${ }^{[23,24]}$. Early studies found that the AUC of CLR increased when omeprazole was given simultaneously with clarithromycin ${ }^{[7]}$, whereas only the $T_{\max }$ was increased with lansoprazole coadministration $^{[8]}$. In our study, an increased $C_{\max }$ of CLR was the only alteration observed after combination therapy, and this effect was likely a result of increased absorption due to the effect of ilaprazole on gastric $\mathrm{pH}$ elevation ${ }^{[10,11]}$. Because clarithromycin tends to be degraded into an inactive form in low $\mathrm{pH}$ environments, one possible reason for the observed synergy was due to the higher bioavailability of clarithromycin in low hypoacidic environments ${ }^{[10,11]}$. Therefore, PPIs may enhance the absorption of clarithromycin due to a sustained elevation of $\mathrm{pH}^{[12]}$.

Our study did not measure ilaprazole concentrations in urine, which may have given us direct evidence of the bioavailability of this compound. However, we observed a decline in the major metabolite of ilaprazole after simultaneous administration with clarithromycin and amoxicillin, which indicates that the decrease in ilaprazole absorption is due to another mechanism. Thus, the urine concentration of clarithromycin should be analyzed in further studies to provide more evidence for this hypothesis.

In summary, our study reveals a drug interaction between a new proton pump inhibitor, ilaprazole, and clarithromycin after triple drug combination therapy. We observed a decrease of ilaprazole and a slight increase of clarithromycin. However, these effects were not dose-dependent and did not affect the therapeutic effects of these drugs.

\section{Acknowledgements}

We would like to thank Mary W ROEDERER, PharmD, BCPS, at the UNC Eshelman School of Pharmacy, Chapel Hill, NC, USA, for her contribution to the preparation of this manuscript.

This work was supported by the National Natural Science Foundation of China (№ 30801421, 30901834, and 30873089), the Huge Project to Boost Chinese Drug Development, (№ 2009ZX09501-032), the 863 Project (№ 2009AA022710, 2009AA022703, and 2009AA022704), the New Century Excellent Talents Project (NCET-10-0843) and Funda- 
mental Research Funds for the Central Universities (No 2010QZZD010).

\section{Author contribution}

Shan CAO, Dong-sheng OU-YANG, Dong GUO, Lan FAN, Hong-hao ZHOU, and Wei ZHANG designed research; Shan CAO, Gan ZHOU, Hui-zi WU, Kui XIAO, Yao CHEN, and Zhi-rong TAN performed research; Hai-tang HU and Xianghong QIN contributed new reagents or analytic tools; Shan CAO, Dong-sheng OU-YANG, and Wei ZHANG analyzed data; Shan CAO, Lan FAN, and Wei ZHANG wrote the paper.

\section{Supplementary information}

Supplementary table (Table 1S) is available at the Acta Pharmacologica Sinica website.

\section{References}

1 Pounder RE, Ng D. The prevalence of Helicobacter pylori infection in different countries. Aliment Pharmacol Ther 1995; 9: 33-9.

2 Malfertheiner P, Megraud F, O'Morain C, Hungin AP, Jones R, Axon $A$, et al. Current concepts in the management of Helicobacter pylori infection - the Maastricht 2-2000 Consensus Report. Aliment Pharmacol Ther 2002; 16: 167-80.

3 Peterson WL, Graham DY, Marshall B, Blaser MJ, Genta RM, Klein PD, et al. Clarithromycin as monotherapy for eradication of Helicobacter pylori: a randomized, double-blind trial. Am J Gastroenterol 1993; 88: 1860-4.

4 Hirschl AM, Rotter ML. Amoxicillin for the treatment of Helicobacter pylori infection. J Gastroenterol 1996; 31: 44-7.

5 van der Hulst RW, Keller JJ, Rauws EA, Tytgat GN. Treatment of Helicobacter pylori infection: a review of the world literature. Helicobacter 1996; 1: 6-19.

6 Lamouliatte H, Cayla R, Zerbib F, Forestier S, de Mascarel A, JoubertCollin $\mathrm{M}$, et al. Dual therapy using a double dose of lansoprazole with amoxicillin versus triple therapy using a double dose of lansoprazole, amoxicillin, and clarithromycin to eradicate Helicobacter pylori infection: results of a prospective randomized open study. Am J Gastroenterol 1998; 93: 1531-4.

7 Calabresi L, Pazzucconi F, Ferrara S, Di Paolo A, Tacca MD, Sirtori C. Pharmacokinetic interactions between omeprazole/pantoprazole and clarithromycin in health volunteers. Pharmacol Res 2004; 49: 493-9.

8 Mainz D, Borner K, Koeppe P, Kotwas J, Lode H. Pharmacokinetics of lansoprazole, amoxicillin and clarithromycin after simultaneous and single administration. J Antimicrob Chemother 2002; 50: 699-706.

9 Hassan-Alin M, Andersson T, Niazi M, Liljeblad M, Persson BA, Rohss K. Studies on drug interactions between esomeprazole, amoxicillin and clarithromycin in healthy subjects. Int J Clin Pharmacol Ther 2006; 44: 119-27.

10 Gustavson LE, Kaiser JF, Edmonds AL, Locke CS, DeBartolo ML, Schneck DW. Effect of omeprazole on concentrations of clarithro- mycin in plasma and gastric tissue at steady state. Antimicrob Agents Chemother 1995; 39: 2078-83.

11 Unge P, Andersson T. Drug interactions with proton pump inhibitors. Drug Saf 1997; 16: 171-9.

12 Ateshkadi A, Lam NP, Johnson CA. Helicobacter pylori and peptic ulcer disease. Clin Pharm 1993; 12: 34-48.

13 Ushiama H, Echizen H, Nachi S, Ohnishi A. Dose-dependent inhibition of CYP3A activity by clarithromycin during Helicobacter pylori eradication therapy assessed by changes in plasma lansoprazole levels and partial cortisol clearance to 6beta-hydroxycortisol. Clin Pharmacol Ther 2002; 72: 33-43.

14 hHo KY, Kuan A, Zano F, Goh KL, Mahachai V, Kim DY, et al. Randomized, parallel, double-blind comparison of the ulcer-healing effects of ilaprazole and omeprazole in the treatment of gastric and duodenal ulcers. J Gastroenterol 2009; 44: 697-707.

15 Wang L, Zhou L, Lin S, Hu H, Xia J. A new PPI, ilaprazole compared with omeprazole in the treatment of duodenal ulcer: a randomized double-blind multicenter trial. J Clin Gastroenterol 2011; 45: 322-9.

16 Zhou G, Shi S, Zhang W, Tan Z, Chen Y, Guo D, et al. Identification of ilaprazole metabolites in human urine by HPLC-ESI-MS/MS and HPLCNMR experiments. Biomed Chromatogr 2010; 24: 1130-5.

17 Cho H, Choi MK, Cho DY, Yeo CW, Jeong HE, Shon JH, et al. Effect of CYP2C19 genetic polymorphism on pharmacokinetics and pharmacodynamics of a new proton pump Inhibitor, Ilaprazole. J Clin Pharmacol 2012; 52:976-84.

18 Li Y, Zhang W, Guo D, Zhou G, Zhou H, Xiao Z. Pharmacokinetics of the new proton pump inhibitor ilaprazole in Chinese healthy subjects in relation to CYP3A5 and CYP2C19 genotypes. Clin Chim Acta 2008; 391: 60-7.

19 Zhou G, Tan ZR, Zhang W, Ou-Yang DS, Chen Y, Guo D, et al. An improved LC-MS/MS method for quantitative determination of ilaprazole and its metabolites in human plasma and its application to a pharmacokinetic study. Acta Pharmacol Sin 2009; 30: 1330-6.

20 Shimizu M, Uno T, Yasui-Furukori N, Sugawara K, Tateishi T. Effects of clarithromycin and verapamil on rabeprazole pharmacokinetics between CYP2C19 genotypes. Eur J Clin Pharmacol 2006; 62: $597-$ 603.

21 Londong W, Gorgas R, Pommerien W, Marsch-Ziegler U, Semmler $\mathrm{P}$, Rost KL, et al. Effect of different omeprazole doses combined with amoxicillin on intragastric $\mathrm{pH}$, amoxicillin bioavailability and Helicobacter pylori eradication in duodenal ulcer patients. Gastroenterology 1995; 108: A153.

22 Pommerien W, Braun M, Idström JP, Wrangstadh M, Londong W. Pharmacokinetic and pharmacodynamic interactions between omeprazole and amoxicillin in Helicobacter pylori-positive healthy subjects. Aliment Pharmacol Ther 1996; 10: 295-301.

23 Goddard AF, Jessa MJ, Barrett DA, Shaw PN, Idstrom JP, Cederberg C, et al. Effect of omeprazole on the distribution of metronidazole, amoxicillin, and clarithromycin in human gastric juice. Gastroenterology 1996; 111: 358-67.

24 Ferrero JL, Bopp BA, Marsh KC, Quigley SC, Johnson MJ, Anderson DJ, et al. Metabolism and disposition of clarithromycin in man. Drug Metab Dispos 1990; 18: 441-6. 